\title{
A Reflection on Using Face-to-Face Peer ReView as a Method of Providing Formative FEedBACK
}

\author{
Jeffrey Harris, Julia Filiplic, Hema Nookala, and Nicholas Petrelli \\ University of Toronto \\ jharris@mie.utoronto.ca
}

\begin{abstract}
Face-to-face peer review was introduced into a third-year engineering course as a mechanism for providing formative feedback on written reports and oral presentations.

The design of the peer review exercises are a work-inprogress, and in this paper we present our reflections on the first experience of using peer review in this course. As authors, we are the course lecturer and three students, and so we present our reflections from both instructor and student perspectives.

Through our reflections, we identified that peer review was a valuable tool for formative feedback. We suggested that student engagement could be increased by improving the structure how peer review was implemented in the course.
\end{abstract}

Keywords: Peer review, Feedback, Engineering reports, Oral presentations, Engineering communication

\section{INTRODUCTION}

Peer review, also called peer evaluation or peer assessment, is the process by which students give feedback on the work of other students. One of the benefits of peer review is that it allows the opportunity for greater amount of formative assessment: that is, the evaluation of learning as it is happening. This forwardlooking type of assessment fosters continual improvement.

Peer review benefits both the assessee and the assessor. By receiving constructive feedback, the assessee has the opportunity to use that feedback to improve his/her own work. By critically evaluating the work of others, the assessor develops transferrable skills that can help him/her improve his/her own work [1]. Acting as an assessor has been shown to improve metacognition and self-assessment [2].

In engineering education, peer review processes have been used for providing feedback on written work $[3,4]$ and oral presentations [5]. In these cases, students gave each other feedback in the written form. Written feedback provides minimal opportunity for the assessors to ask questions and for the assessees to clarify the meaning of comments they receive. On the other hand, in other disciplines educators have found that face-to-face peer review promotes discovery mode feedback by providing the opportunity for probing [6]. This means that face-toface review allows for the assessor and assessee to construct a mutual understanding. In addition, using a face-to-face review allows students to get their feedback quicker than they would if written feedback was used.

In a third-year 'Design for Environment' course with approximately 200 students at the University of Toronto, we introduced face-to-face peer review for a team-written engineering report and an oral presentation. This endeavour is a work-in-progress: we implemented peer review for the first time in the winter 2017 term, and we are in the process of reflecting on the experience so that we can improve for the next iteration.

This paper is written jointly by the course lecturer (Jeffrey) and three students who took the course as a student (Julia, Hema, and Nicholas). Therefore, we present reflections from both instructor and student perspectives.

\section{IMPLEMENTATION}

\subsection{Purpose of Peer Review}

We implemented peer review activities only as exercises for formative feedback. This allowed students to use the peer review to improve their work before they submitted or presented a final version for evaluation.

In our implementation, the purpose of peer review was to facilitate improvement through feedback and iteration. This meant that we did not use peer review as mechanism to assign grades to students. The students' grades were entirely assessed by teaching assistants and the course lecturer.

\subsection{Peer Review of Written Work}

A peer review of a written engineering report was conducted as an in-class exercise during a 50-minute lecture. This lecture was jointly facilitated by the course lecturer (Jeffrey Harris) and a teaching assistant. In the 
first 25 minutes of the lecture, students were given instruction on how to critically evaluate the work of others using a rubric. This was done through an example: students were given 5 minutes to read an excerpt from a report written by students in a prior year. The teaching assistant then walked through the excerpt, explaining the feedback he would give the authors and how he would evaluate the report using the project rubric. The lecturer elaborated by describing ways of structuring comments so that they provide constructive feedback.

In the subsequent 25 minutes, students participated in a reciprocal face-to-face peer review of their own written reports. In advance, students were asked to print one section of their draft report and bring it to lecture. In class, each student exchanged a draft with a peer. Students were asked to spend 5 to 10 minutes reading the work of their peers and then spend the remainder of the time giving each other feedback. The course lecturer and teaching assistant circulated through the classroom and interacted with pairs of students.

After the in-class peer review, students had two days to complete and modify their written work before submitting it to their teaching assistant for grading.

\subsection{Peer Review of Oral Presentations}

As a non-graded formative exercise, student teams rehearsed an oral presentation in front of an audience consisting of a teaching assistant and one or two other student teams. Teaching assistants were encouraged to facilitate face-to-face peer feedback immediately after the presentation rehearsal. In practice, each teaching assistant used peer feedback differently, and some teaching assistants opted to give students feedback directly. After the formative rehearsal, students were able to modify their presentation before giving a final (evaluated) presentation to the course lecturer or head teaching assistant.

\section{SELF-REFLECTION}

Each co-author independently reflected on his/her lived experience with peer review in Design for the Environment, and these reflections are each presented in a separate subsection. The subsection title identifies the first name of the author and his/her role in the course.

\subsection{Jeffrey (Course Lecturer)}

3.1.1 Peer Review of Written Work. I decided to facilitate a peer review for the written report because I wanted students to better understand the project requirements and the difference between a report that "meets expectations" and a report that "exceeds expectations." In the previous iteration of the course, quite a number of students were disappointed with their evaluation, and through conversations with those students, I sensed that they focused on the descriptors in the "exceeds expectations" rubric column (without thoughtfully reading the descriptors in other columns). Therefore, in the current iteration of the course, I added an in-class peer review exercise so that students would practice interacting with the rubric as an assessor. This practice would develop their ability to self-assess, and write better reports.

I decided to conduct the peer review of written reports as an in-class exercise because I did not want to add to the students' workload (outside of class hours). The Design for Environment course is part of a very demanding term for students, and so I was very hesitant to increase the amount of work they had to complete outside of class. With this in mind, students were not asked to submit anything before class: they were only asked to bring a draft (of one section of their report) to class.

At the start of the peer review lecture, it was apparent that a small number of students were unprepared. Some students left the class for a few minutes so that they could print their report in a computer lab, and other students choose to pass their laptop to their assessor (instead of bringing a printed copy to class). During the peer review activity, however, students were actively engaged. As I circulated the classroom, I saw students focused on their task, and I overheard students giving each other meaningful feedback. I did not see any distracted students in class. After the class had finished, I found it difficult to measure the outcome of the peer review exercise. Since students gave each other verbal feedback face-to-face, I was unable to assess how students were using the rubric and the quality of the comments they gave each other.

3.1.2 Peer Review of Oral Presentations. I encouraged teaching assistants to facilitate the exchange of verbal peer feedback during presentation rehearsals, but unfortunately, not all teaching assistants used peer review.

In prior years, presentation rehearsals were done privately with only the presenting group and the teaching assistant. In the previous iteration of the course, many students were unprepared and had incomplete presentations at their rehearsal. In the current iteration of the course, I reconfigured the rehearsals so that students would be rehearsing in front of some peers (in addition to the teaching assistant). This gave the students a larger audience, providing them an incentive to be better prepared at their rehearsal. The inclusion of peer feedback at the rehearsal was intended as a mechanism to actively engage the students in the audience and create valuable learning experience.

The peer review was not fully implemented due to two main factors: time management and insufficient teaching assistant training. Scheduling forty-eight 12-minute 
presentation rehearsals in the span of three days was a logistic challenge when student availability, teaching assistant availability, and room availability were accounted. In many cases, this left little time for students to give each other meaningful feedback or ask probing questions. In such cases, the teaching assistants often opted to be time-efficient by giving feedback directly (i.e. TA-to-student). In a future iteration of the course, I would ideally allow more time for students give each other feedback. If I had to work with the same time constraints as this year, I would consider using written peer feedback instead of a face-to-face discussion.

Insufficient teaching assistant training was a barrier because not all teaching assistants were comfortable taking on a facilitator role. In a future course iteration, I would offer teaching assistants some guidance of facilitating a discussion.

\subsection{Julia (Student)}

I found that having the opportunity to give and receive feedback from my peers in Design for the Environment helped me to think about my abilities to present engineering communication challenges, such as forming strong arguments, structuring reports in a clear manner, and justifying design decisions.

When looking at other students documents in the inclass peer review session, it gave me insight into how others were approaching engineering requirements such as deciding how to present information, what they decided to include, assumptions they made, and how they justified those and other decisions. It allowed me to think about those reasons for myself as well and gave me the chance to compare work without being given an 'outline' or something to explicitly model work after. This was especially useful in Design for the Environment: each group was working on a different topic for their project, and so I don't think that being given a template or exact instructions on how to approach the final project would've made sense because it might not have applied to everyone. Having both the professor and teaching assistant present was incredibly helpful as well because I could ask what sort of things they look for when grading, putting myself in that position, which made me think about those same things later when working on my report.

However, I think that for a greater number of students to get these and other benefits of a peer review, preparation and planning are key. Having students come to class with their work printed out, maybe different coloured pens, and knowing exactly what they're doing and how they can benefit so that they make sure to come to class. I also could have benefitted from this more if I had perhaps been pushed to follow up with it more; it's easy to forget about one useful activity from a few weeks ago when you've been working in the lab all weekend and are mostly focused on meeting the deadline. I think it would have been fantastic to have a student from outside my group take a look at our report periodically during the term so that a fresh pair of eyes can read things over and give new perspective on it.

Rehearsing presentations in front of other groups was fantastic, especially because I knew my group would be presenting in front of groups for the final presentation, so it gave me a sense of what the atmosphere would be like. My peers were supportive and gave good feedback, and our TA let us speak before him so I felt like the groups were taking in the feedback. Everyone has different skills, abilities, and levels of competence, so having the opportunity to share strengths or get feedback from a variety of people I think can be more valuable than just one person. It's possible to have a group with members who haven't had much presentation experience, so they would benefit from feedback from another group whose members have had more experience.

I also think that the sooner students can start reviewing each other's work in their undergraduate career (or even earlier), the more time they have to develop these skills and how to give constructive feedback to others about them. I think students are sometimes scared to get critiques from others because they don't want to think there's anything 'wrong' with their work, or because they've been doing something for a while they may not want to change or alter how they do it. Overall though, I think peer review fits into the design processes of engineering by iterating, constantly improving, and thinking about next steps: something all engineering students should value.

\subsection{Hema (Student)}

When I was told that we would have a peer feedback session for our preliminary consultant report (PCR), I was honestly excited. Feedback isn't provided very often during university and instructions are often vague or can be easily misinterpreted. So an opportunity for feedback, even if it wasn't from anyone related to the marking of this project was wholly welcome. However, if I remember correctly, there were a few problems that made it such that our team did not get as much out of it as we could. Firstly, I don't think our document was written fully enough that we could get feedback on the things our peers could help us on (grammar, structure, setup, appearance, logic). Secondly, there weren't very many people physically at the lecture, which decreased the pool of potential feedback. However, I still believe that pre-submission feedback could be very useful. As a suggestion for an alternate structure, teams could have a deadline to upload their document online and after that deadline each student must virtually markup at least one PCR other than 
their own (maybe set up a matching system to each team's PCR will get marked up). But making these things mandatory and able to do in the comfort of our own homes would definitely increase the range of effect. This way each team will have feedback from a variety of people and may be better prepared for their final submission. All in all, university needs to have more venues where students can submit their work and get legitimate feedback, whether that be before or after receiving a mark. Constant feedback is the only way to constantly improve, which is what the real world requires from us.

\subsection{Nicholas (Student)}

Student interaction during lecture is an important part of learning, you only absorb a certain amount of information while the professor is speaking. Actually talking to your peers and discussing concepts reinforces what was taught, and can give you different perspectives and ways to look at the information.

Discussing our PCR project with other groups allowed each group to get feedback from peers on their project. This benefitted me, as having my work critiqued would ensure that I have done a good job, and by reading through a peers work, would allow me to see a different way to portray the information or thoughts.

A way to improve this would to add more structure to the feedback system, many students did not bring a copy of their work, or take the exercise seriously. This can be added as a mandatory part of the design process in a course, as a major part of being an engineer is the ability to collaborate and give feedback to different team members/colleagues.

The feedback was useful, as any criticism is good criticism. I was prepared for the activity by bringing a printed copy of my PCR, but many were not. I used the exercise seriously, as there were parts of my PCR that I was unsure about, so reading over another groups part, gave me insight on how to tackle those sections.

\section{INTERPRETATION \& NEXT STEPS}

Overall, all four co-authors felt that peer review was a worthy exercise because it facilitated iterative improvement of student work. The three students (Julia, Hema, and Nicholas) identified that the feedback from their peers was valuable. Julia and Nicholas further stated that they were able to improve their own work as an effect of evaluating the work of other students.
All four co-authors identified that some students were unprepared for the peer review of the written report and highlighted that student preparedness could be encouraged by revising the structure of the peer review. For a different workshop in the course, students were required to submit a completed worksheet in advance of the workshop. Although the worksheet was not graded, almost all of the students submitted the worksheet because it had a 'required' deadline. Similarly, 'requiring' students to submit a draft report (in advance of the peer review lecture) could increase student engagement in class. Hema also suggested that online written feedback might be an alternative mode that could increase student engagement.

As a student, Julia felt that the peer review could be enhanced with follow-up activities, and as an instructor, Jeffrey desired a mechanism to evaluate the effectiveness of the peer review exercises. These two demands could potentially be addressed by asking students to formally (but briefly) reflect on how they used peer feedback in their editing process.

None of the authors reflected specifically on the faceto-face mode of delivering peer feedback. Therefore, in this preliminary work, we are unable to evaluate the effectiveness of the face-to-face mode and the value of asking follow up questions during a peer review. This question would be valuable to probe in a subsequent iteration.

\section{References}

[1] K. J. Topping, E. F. Smith, I. Swanson, and A. Elliot, Assessment \& Evaluation in Higher Education, 25(2), 149169 (2000)

[2] K. J. Topping, Theory Into Practice, 48, 20-27 (2009)

[3] P. M.Ostafichuk, C. P. Jaeger, J. Nakane, S. Nesbit, N. Ellis, and J. Sibley, Proc. 2016 Canadian Engineering Education Association Conf. (2016)

[4] M. Orjuela-Laverde and L. R. Chen, Proc. 2014 Canadian Engineering Education Association Conf. (2014)

[5] J. Lax and A. W. Fentiman, 2016 ASEE Annual Conference \& Exposition, Paper \#14691 (2016)

[6] J. M. Crossman and S. L. Kite, Active Learning in Higher Education 13(3), 219-229 (2012) 